\title{
Die Medizin: Auf der Suche nach einem neuen Menschenbild
}

\author{
Piet van Spijk \\ Dr. med. und Dr. phil., Facharzt für Allgemeine Innere Medizin, Mitglied FMH, Präsident Forum Medizin und Philosophie
}

\begin{abstract}
Am Ursprung des Erfolgs der westlichen Medizin standen nicht technische Neuentwicklungen oder wissenschaftliche Entdeckungen. Es war vielmehr ein neues Menschenbild. Dieses wurde von René Descartes (1596 bis 1650) ausformuliert und bestand im Wesentlichen darin, den Menschen als zweigeteilt zu verstehen - bestehend aus einem Körper auf der einen und einem Geist auf der anderen Seite.
\end{abstract}

\section{Das Maschinenmodell des Menschen}

Während für Descartes die Beziehung zwischen Körper und Geist unklar blieb, lösten Autoren wie Julien Offray de La Mettrie (1709-1751) das Problem dieser unklaren Beziehung, indem sie beide - Körper und Geist gleichermassen als etwas Physisch-Materielles verstanden (sog. physikalischer Monismus). Sie sahen den Menschen als eine physikalischen Gesetzen gehorchende Maschine, und so heisst denn auch das Hauptwerk La Mettries «L'homme machine».

Erst zwei Jahrhunderte später wurde die Konzeption des «Menschen als Maschine» zum allgemein akzeptierten Gedankengut nicht nur bei Wissenschaftlern, sondern auch bei Ärzten und Patienten. Die wissenschaftlichen Erkenntnisse und daraus abgeleiteten medizinischen Erfolge, die sich ab dem 20. Jahrhundert auf der Grundlage der Maschinen-Theorie des Menschen einstellten, brachten diesem Denken den Durchbruch. Unterdessen ist es üblich geworden, das Herz als Pumpe und die Nieren als Kläranlage zu verstehen. Und ganz im Sinne von La Mettrie gilt heute der Computer als Modell für das Gehirn mit all seinen geistigen und emotionalen Ausdrucksformen.

\section{Nachdenken über zentrale Themen der Medizin}

Die Medizin sieht sich gegenwärtig einer Reihe von Problemen ausgesetzt, die im Laufe der letzten Jahre an Virulenz eher zu- als abgenommen haben. Oft wird moniert, für nachhaltige Lösungen fehle es am politischen Willen. Das «Forum Medizin und Philosophie», eine Gruppe philosophisch interessierter Ärzte (Mitglieder des Forums Medizin und Philosophie: www.fomep.ch), möchte die Hypothese stark machen, dass es mindestens ebenso sehr an den ungenügenden theoretischen Grundlagen liegt, auf welchen die heutige Medizin ihre Praxis aufbaut.

Mit Artikeln wie dem vorliegenden möchte das Forum zu vertieftem Nachdenken über zentrale Themen in der Medizin einladen. Dies ist mit der Überzeugung verbunden, dass die erfolgreiche Zukunft der westlichen Medizin in grossem Masse davon abhängt, ob es gelingt, die konzeptuellen Grundlagen der heutigen Medizin neu zu denken, allgemein verständlich zu beschreiben und in offener Diskussion in ihren Konturen zu schärfen. Es wäre schön, wenn sich die Leserschaft der SÄZ an dieser Diskussion beteiligte. (pvs)
Trotzdem wird es immer offensichtlicher, dass die Reduktion des Menschen auf ein berechenbares Maschinenmodell den Anforderungen einer zukunftsträchtigen Medizin nicht mehr gerecht zu werden vermag. Die komplexen Multimorbiditäten, welche heute die grosse therapeutische Herausforderung darstellen, lassen sich physikalistisch-mechanistisch nur mangelhaft verstehen. Viele Patienten akzeptieren es zu Recht nicht mehr, als Behandlungsgegenstände (die es zu reparieren gilt) und nicht als einmalige und unverwechselbare Individuen behandelt zu werden. Auch auf theoretischer Ebene wird klar, dass dieses Modell mangelhaft, wenn nicht sogar falsch ist. Neben der Tatsache, dass Maschinen selbst einfachen Lebewesen an Komplexität um Dimensionen unterlegen und vollständig von Menschen abhängig sind, ist es offensichtlich, dass Maschinen weder wachsen noch sich entwickeln oder sich fortpflanzen können und dass sie-als entscheidendes Element - ohne subjektives Erleben sind; sie kennen weder einen eigenen Antrieb noch Schmerzen oder Gefühle wie Angst oder Liebe.

Aus diesen Erkenntnissen heraus wurde im Laufe des 20. Jahrhunderts das Maschinen- (auch bio-medizinisches Modell) zum bio-psycho-sozialen Modell des Menschen erweitert.

Die Vertreter dieses Menschenbildes sprechen davon, dass es heute die bedeutendste Theorie für die Beziehung zwischen Körper und Geist sei, erstmals ein wissenschaftlich begründetes, ganzheitliches Verständnis von Krankheit bzw. Gesundheit erlaube und insbesondere auch der WHO als Basis ihrer Arbeit diene. [1, 2] Doch sie reihen die Bereiche des Biologischen, Geistigen, Psychischen und Sozialen einfach zusammenhangslos aneinander, ohne angeben zu können, wie sie in Beziehung zueinander stehen. Sie gehen wie Descartes davon aus, dass das Biologische auf der einen Seite 
seinen festen Platz in Raum und Zeit hat und den Gesetzen der Physik und der Chemie unterliegt und dass das Psychisch-Geistige auf der anderen Seite weder Raum noch Zeit noch Stofflichkeit kennt. Doch für diese beiden grundsätzlich unterschiedenen Bereich gilt: Was nichts miteinander gemein hat, kann auch nicht in Kontakt treten, ist und bleibt unverbunden. Denn mindestens die beiden Kontaktflächen müssen, sich angleichend und anschmiegend, gegenseitig ähnlich sein.

Zeitgenössische Modelltheoretiker versuchen das Problem dadurch zu lösen, dass sie behaupten, Körper und Geist-Seele würden eine Einheit bilden. [3] Dieser Lösungsversuch läuft deshalb ins Leere, weil die beiden Bereiche in ihrer grundsätzlichen Unterschiedlichkeit definitionsgemäss nicht eins sein können.

So sehen sich die Vertreter des bio-psycho-sozialen Menschenbilds mit demselben Problem konfrontiert wie vor fast 400 Jahren René Descartes: Die Beziehung zwischen Körper und Seele oder zwischen vermessbaren, physikalisch-chemischen Gesetzen gehorchenden Hirnfunktionen und subjektivem, sich der präzisen wissenschaftlichen Untersuchung entziehendem Geist bleibt unüberwindbar. Das bio-psycho-soziale Menschenbild wird so unserem Bedürfnis nach Kohärenz und Sinnhaftigkeit nicht gerecht.

\section{Dringend gesucht: ein neues Menschen- bild für die Medizin}

Wer immer sich für diese Art von Gedankenarbeit und Reflexion interessiert und sich dazu zusätzliche Kenntnisse aneignen möchte, den dürften die folgenden Links interessieren: https://fomep.ch/ https://www.unilu.ch/ weiterbildung/ksf/ cas-philosophie-undmedizin/ http://www.weiterbil dung.uzh.ch/programme/ detail.php?angebnr $=43$ https://advancedstudies. unibas.ch/studienange bot/kurs/cas-philosophiefuer-fachleute-aus-medi zin-und-psychotherapiein-planung-19761

Korrespondenz:

Piet van Spijk MEDICUM WESEMLIN

Landschaustrasse 2

CH-6006 Luzern

piet.vanspijk[at]medicum-

wesemlin.ch
Mehr als neue Onkologika, Biologika, Gefässdevices, mehr als neue PETs und Operationsroboter braucht die Medizin als Handlungsgrundlage ein Menschenbild, in welchem sich erstens der Laie wiedererkennt, welches zweitens den medizinischen Aktivitäten sinnvolle Ziele vorgibt und das drittens den heute überhandnehmenden Fehlentwicklungen (Kommerzialisierung, Fragmentierung der Patientenbetreuung, Medikalisierung des Alltags etc.) entgegenwirkt. Dass eine geeignetere Modellvorstellung des Menschen in der medizinischen Praxis noch auf sich warten lässt, hat möglicherweise damit zu tun, dass wir, von Alltagssorgen getrieben und von technischen Neuerungen geblendet, etwas Wesentliches verpassen: das Nachdenken über den Menschen! Wichtige Sackgassen beim Nachdenken über den Menschen wurden in den obigen Ausführungen bereits erwähnt:

Es gilt erstens zu vermeiden, ihn mit einem Gegenstand (z.B. mit einer Maschine) zu verwechseln. Das ist oft leichter gesagt als getan, ist doch selbst der Bau untantive - mit ihren Eigenschaften - den Adjektiven - zu bezeichnen. [4] In der Wissenschaft gibt es Forschungsobjekte, keine Forschungssubjekte. Dasselbe gilt für serer Sprache darauf ausgelegt, alles als Dinge - Subs-
Qualitätsindikatoren in der Medizin, wo die subjektive Meinung des Patienten gerne ausgeklammert wird.

Problematisch ist es auch, den Menschen als System zu verstehen. Das Systemkonzept ist im Rahmen der Steuerung von Maschinen entstanden und hat es nie geschafft, diesen Bezugsrahmen hinter sich zu lassen. Vor allem das subjektive Erleben von Lebewesen in Form von Gefühlen, Motivation und Wille findet im Systemdenken keinen gebührenden Platz.

In einem neuen und zukunftsträchtigen Verständnis des Menschen ist zweitens die Unterscheidung von Körper und Seele obsolet. Menschen haben keinen Körper. Eher sind sie Körper. Und selbst diese Aussage ist verwirrend oder sogar falsch. Menschen sind Organismen und sind als solche bezüglich Organisation, Komplexität und Eigenaktivität von Körpern und von Dingen fundamental verschieden. Um einer verhängnisvollen Verwechslung vorzubeugen, wäre es wohl ratsam, den Begriff des «Körpers» den leblosen Dingen wie beispielsweise «Zangen", "Maschinen» oder "Betonpfeilern" vorzubehalten.

Menschen haben auch keine Seele. Sie fühlen, denken, sprechen, freuen sich und leiden. Daraus zu folgern, sie hätten ein Etwas - «Seele» genannt -, das fühlt, denkt, spricht etc., entbehrt der Empirie.

Ein neues Menschenbild wird drittens nichts Endgültiges und Feststehendes sein, sondern wird immer wieder neue Entwicklungen und Erkenntnisse aufnehmen und integrieren. Es wird wie alles Leben und daher auch wie alles menschliche Leben in einem ständigen Werden begriffen sein. Es wird in den Vordergrund rücken, dass sich der Mensch immer auf ein Ziel hin ausrichtet, wird den Prozesscharakter des (menschlichen) Lebens betonen und zeigen, dass gewisse Prozesse so langsam ablaufen, dass der Eindruck entsteht, sie stünden still und es gebe so etwas wie feste Strukturen.

Damit sind einige Elemente, welche es bei der Ausarbeitung eines neuen Menschenbildes zu beachten gilt, genannt. Es wird jetzt darum gehen, dieses zu ergänzen, zu präzisieren und breit zu diskutieren. Die Leserschaft der SÄZ ist eingeladen, bei dieser wichtigen Grundlagenarbeit mitzutun.* Jeder Beitrag beispielsweise in Form eines Leserbriefes kann ein unverzichtbarer Schritt in die richtige Richtung sein!

Literatur

1 Egger JW. Das biopsychosoziale Krankheitsmodell; http://www.psygraz.at/fileadmin/user_upload/Psy_2/ Psy201bpsMod2005_Egger.pdf

2 International Classification of Functioning, Disability and Health (ICF)http://www.who.int/classifications/icf/en/

3 Egger JW. Theorie und Praxis der biopsychosozialen Medizin, S. 25f.

4 Sog. Substanz- oder Dingontologie z.B.: http://www.topowiki.de/ wiki/Dingontologie 